\title{
Ethnologies
}

\section{Imaginaire touristique et authenticité à Fès et Istanbul}

\section{Muriel Girard}

Volume 32, numéro 2, 2010

Tourisme culturel

Cultural Tourism

URI : https://id.erudit.org/iderudit/1006305ar

DOI : https://doi.org/10.7202/1006305ar

Aller au sommaire du numéro

Éditeur(s)

Association Canadienne d'Ethnologie et de Folklore

ISSN

1481-5974 (imprimé)

1708-0401 (numérique)

Découvrir la revue

Citer cet article

Girard, M. (2010). Imaginaire touristique et authenticité à Fès et Istanbul. Ethnologies, 32(2), 59-80. https://doi.org/10.7202/1006305ar

\section{Résumé de l'article}

L'article interroge l'authenticité fabriquée par les guides touristiques et, en retour, les impressions des touristes, pour enfin questionner les ambivalences identitaires liées au tourisme. L'étude montre ainsi l'attitude contrastée des guides, entre recours aux stéréotypes associés à l'Orient à Fès et tentative de changer les représentations des touristes pour proposer d'autres versions de l'authenticité à Istanbul. Dans ce contexte, l'artisanat apparaît comme une figure équivoque de la mise en scène de l'authenticité. Mais dans un cas comme dans l'autre, l'imaginaire véhiculé par le guide ne converge qu'imparfaitement avec l'expérience vécue des touristes, basée sur des émotions multiples.
Ce document est protégé par la loi sur le droit d'auteur. L'utilisation des services d'Érudit (y compris la reproduction) est assujettie à sa politique d'utilisation que vous pouvez consulter en ligne.

https://apropos.erudit.org/fr/usagers/politique-dutilisation/ 


\title{
IMAGINAIRE TOURISTIQUE ET AUTHENTICITÉ À FÈS ET ISTANBUL
}

\author{
Muriel Girard \\ Laboratoire CETOBAC, Ecole des Hautes Etudes en Sciences Sociales, Paris
}

\begin{abstract}
« Nous, les guides, nous sommes là pour faire de la culture ${ }^{1}$. « C'est intéressant de partager, mais un guide, durant une semaine, joue un rôle. On ne peut pas descendre de la scène, il n'y a pas de lien $»^{2}$. Ces propos de guides stambouliotes, qu'il convient d'interroger, illustrent le double rôle du guide, de médiateur culturel et de représentation (Chabloz et Raout 2009: 13). Ils donnent aussi à voir les «actions entreprises par des sociétés se construisant ou se reconstruisant avec et à travers le tourisme » (Doquet et Le Menestrel $2006: 3$ ) pour répondre au « regard touristique »(Urry 1990). Quel est alors l'imaginaire touristique mis en scène et véhiculé par le guide?

Interroger l'imaginaire touristique conduit à observer l'authenticité fabriquée par le guide, et, en retour les impressions vécues par les touristes. Malgré le fait que Dennison Nash (1981) ait perçu leur importance, peu d'études ont porté sur les guides touristiques (Chabloz et Raout 2009; Doquet 2009). Les travaux d'Erik Cohen (1985, 2001) ont contribué à préciser leur rôle. Il l'a ainsi défini à partir d'une «fonction communicative» consistant à «faire remarquer les points d'intérêts et expliquer les sites mais surtout les interpréter en fonction des expériences et des attentes touristiques » (Doquet 2009: 79). Suite à ses recherches, "quelques articles se sont attachés à redéfinir la question de l'authenticité et le travail du guide en fonction » (Doquet 2009 : 79). La notion d'authenticité, centrale dans le domaine des études touristiques (Saïdi 2010), a donné lieu à différentes conceptualisations,
\end{abstract}

1. Propos d'un guide anglophone et hispanophone, Chambre des guides d'Istanbul, août 2005.

2. Propos d'un guide francophone, exerçant depuis 1975, Chambre des guides d'Istanbul, août 2005. 
depuis les travaux de Dean MacCannell sur la quête d'authenticité3 (1973, 1976). Tom Selwyn (1996) a ainsi distingué «l'authenticité froide »- mise en scène et constituée d'objets à observer renvoyant à la connaissance liée à l'expérience touristique - et "l'authenticité chaude »- reposant sur l'expérience du touriste et associée à sa recherche de solidarité sociale. C'est avec ces deux types d'authenticité que le guide doit composer pour satisfaire ses clients (Doquet 2009). En outre, son rôle a été repensé à partir de la recherche d' "authenticité existentielle» (Wang 1999), qui conduirait le touriste à découvrir son moi intérieur.

Ceci étant dans cette étude, nous souhaitons avant tout analyser les relations touristiques en nous centrant sur les éléments de la culture locale que le guide médiatise et, en retour, questionner les émotions patrimoniales (Poulot 2006) des touristes. Cette approche pourrait être corrélée à celle de "l'authenticité projective » (Saïdi 2010 : 472-473) qui renvoie à un état vécu émotionnellement par les touristes, permettant de croiser l'authenticité de l'expérience et l'originalité de l'objet (Saïdi 2010). Au-delà de l'étude des rapports entre les imaginaires des acteurs endogènes et exogènes, l'entrée choisie pour questionner la culture touristique est aussi une clé de lecture pour saisir certaines ambivalences identitaires auxquelles le tourisme donne lieu ou met au jour.

Les travaux de Cohen (1985, 2001), Wang (1999), Reisinger et Steiner (2006), Doquet (2009) ont en commun d'atténuer le rôle mystificateur des guides touristiques, constat que nous questionnerons à partir des exemples de Fès et d'Istanbul. A Fès, dès les premières années du Protectorat français au Maroc (1912-1956), la préservation de la médina et de sa «couleur locale » est pensée (Lahbil-Tagemouati 2001). Les images et discours sur la tradition et l'authenticité aujourd'hui déployés remontent à cette époque (Girard 2010). Tout au long du XXe siècle, le phénomène touristique ne cesse de prendre de l'ampleur (Otmani 1985) et, aujourd'hui, entre 350000 et 400000 touristes parcourent Fès chaque année 4 . Istanbul a quant à elle connu un essor touristique extrêmement important depuis la fin des années 1970. Dans

3. Pour une analyse critique de la quête d'authenticité, voir Michaud (2001), Brown (1999). Voir aussi Cravatte (2009) et Saïdi (2010) sur la notion d'authenticité dans les études touristiques.

4. Source : Statistiques de la Délégation du tourisme de la région Fès-Boulemane. 
ce contexte est promue, à partir des années 1980, une vision édulcorée de l'Empire ottoman à partir des images stéréotypées de l'Orient (Bartu 2001 ; Eldem 2010). Depuis la fin des années 1990, l'Etat et les opérateurs touristiques privés vendent aussi la «mosaïque » culturelle et religieuse de l'Empire ottoman (Eldem 2010 ; Pérouse 2003). Et, à côté de la valorisation - ponctuelle - du patrimoine ottoman (Pérouse 2003), se diffuse une esthétique anatolienne dans les espaces touristiques. Actuellement, la ville accueille chaque année trois millions de touristes étrangers (Pérouse 2004). A la différence de Fès, où les touristes étrangers sont essentiellement français, à Istanbul la clientèle étrangère est cosmopolite même si elle demeure principalement européenne.

L'analyse croisée de la mise en tourisme des villes de Fès et d'Istanbul montre ainsi que le souci d'acteurs publics et privés de répondre à la recherche d'altérité des touristes s'est traduit par un processus de folklorisation, prenant entre autres la forme d'une orientalisation, jouant d'images stéréotypées associées à l'Orient. Il semble alors pertinent de mettre en perspective ces deux cas puisqu'ils donnent à voir des rapports contrastés des acteurs locaux - en l'occurrence les guides - à leur culture et révèlent les décalages entre « auto-orientalisation » (Eldem 2010: 231) à Fès et tentation de réorienter les représentations des touristes à Istanbul.

Notre réflexion s'appuie sur des entretiens menés auprès de guides officiels au syndicat d'initiative de la région Fès-Boulemane et à la Chambre des guides touristiques d'Istanbul (TURSOB) ${ }^{5}$. Pour saisir le rôle de guide, trois questionnements se sont posés : quelle est la ville présentée par le guide? Dans quelle mesure le discours du guide contribue-t-il à l'enchantement des touristes ? Existe-il des écarts entre son discours touristique et ses propres représentations patrimoniales ${ }^{6}$ ? Nous nous appuyons aussi sur des entretiens menés auprès de touristes

5. Nous avons interrogé une dizaine de guides à Fès et dix à Istanbul. D'après le recensement de 1992, Fès comptait deux cent quarante guides officiels (Adner et Bastin 2000). Selon les chiffres donnés par la Chambre des guides touristiques d'Istanbul, neuf mille guides officient, travaillant sur l'ensemble de la Turquie.

6. Les entretiens s'articulent autour de cinq lignes directrices : le parcours personnel du guide ; le type de touristes qui fait appel à lui ; la ville qu'il fait découvrir ; le type de questions posées par les touristes; ses représentations patrimoniales. 
à Fès et Istanbul ${ }^{7}$. Nous avons cherché à cerner les impressions des touristes sur la ville - celles qu'ils pouvaient avoir avant leur voyage et celles qui se sont forgées suite à leur visite. Pour ce faire, nous les avons interrogés sur le choix de leur destination, leur lecture des guides de voyage, la nature de leur visite sur place et les impressions retenues de leur séjour. Des observations ont aussi été réalisées dans des espaces touristiques.

Nous voudrions proposer une lecture de l'imaginaire touristique en partant du discours des guides touristiques, en montrant dans un premier temps comment les guides cherchent à alimenter ou, au contraire, contrarient le désir d'authenticité des touristes. Nous verrons que, dans ce contexte, l'artisanat apparaît comme une figure équivoque de la mise en scène de l'authenticité. Enfin, nous nous pencherons sur les impressions ressenties par les touristes.

\section{Le guide, passeur culturel et producteur d'authenticité}

Dans quelle mesure les guides élaborent-ils un discours répondant aux désirs de leurs clients, contribuant ainsi à susciter leur enchantement? La production de ce discours induit une compréhension des touristes ainsi qu'une habileté à faire varier "l'authenticité chaude » et «froide» selon les clients (Doquet 2009). Mais, aussi fine que soit leur connaissance des touristes et leur volonté d'adapter leur posture, la malléabilité du discours et de la visite est soumise à des contraintes de temps et au rapport que les guides entretiennent avec les agences de voyage $^{8}$. Les guides officiels à Fès, qui travaillent soit pour des touristes

7. À Fès, les entretiens ont été conduits auprès de touristes voyageant seuls mais aussi en voyages organisés (un cinquième des entretiens réalisés). Les entretiens se sont déroulés, à quelques exceptions près, dans des hôtels et des restaurants. Vingt-deux entretiens, impliquant cinquante et une personnes (en majorité des couples, plus rarement des personnes seules ou des petits groupes) ont été réalisés. Les touristes interrogés sont pour la plupart français (trois-quarts des entretiens réalisés), mais aussi suisses, belges, allemands, canadiens et espagnols. A Istanbul, les entretiens ont été effectués place Sultanahmet, haut lieu touristique de la péninsule historique. Nous avons interrogé des touristes libyens, iraniens, pakistanais, polonais, russes, bulgares, américains, néo-zélandais, italiens, allemands, français, israéliens et anglais. Vingt et un entretiens, impliquant quarante-quatre personnes ont été réalisés.

8. À Fès, la durée moyenne de séjour est de deux jours (Source : Conseil régional du tourisme de la région Fès-Boulemane). A Istanbul, dans le cadre de voyages 
individuels soit pour des groupes ${ }^{9}$, auxquels cas un tour opérateur peut faire appel à eux, paraissent avoir une marge d'action plus grande qu'à Istanbul. Les programmes sont toutefois assez précis et uniformes, alliant monumentalité et activités économiques et offrant une vision stéréotypée de l'Orient réduit à ses souks et ses mosquées. La visite est parfois orientée en fonction de l'envie des clients et du temps imparti, les touristes voyageant en groupe ayant des plannings serrés et des lieux précis à visiter, notamment les bazars.

A Istanbul, l'échange entre le guide et le touriste apparaît limité notamment en raison du manque de temps - un élément évoqué de façon récurrente dans l'ensemble des propos des guides interrogés. La marge de manœuvre des guides est réduite, chaque élément de la visite (les principaux hauts lieux de la péninsule historique) étant chronométré et orchestré par les agences de voyage tandis que le discours des guides touristiques est en partie calibré par celles-ci. Ce n'est que lorsqu'ils exercent parallèlement en indépendants et accompagnent quelques touristes, ce qui est rare, que les guides acquièrent une plus grande autonomie dans la création du cadre de l'expérience.

Le dialogue entre le touriste et le guide, élément de partage ou de distinction

Alors que les questions posées par les touristes apparaissent relativement similaires, les guides à Fès et Istanbul adoptent une attitude différente, entre écoute et rapport de distinction. A Fès, d'après les guides interrogés, les questions des touristes concernent en premier lieu la société au sens large. Elles portent sur l'Islam, sur «ce qu'on pense de l'Islam, comment l'Islam voit l'Occident $»^{10}$, la place de la femme en Islam et les coutumes liées au mariage. Plus généralement, ce premier type de questions concerne les traditions et les modes de vie. Une deuxième série de questions a trait plus spécifiquement à la médina, son histoire et son fonctionnement, notamment le transport à dos d'âne. De la même façon, à Istanbul, les questions relèvent essentiellement du

organisés, la visite d'Istanbul s'échelonne sur une demi- journée, une journée, deux journées mais rarement plus (Source: Chambre des guides touristiques d'Istanbul).

9. La quasi-totalité des touristes interrogés voyageant seuls recourt à un guide, au moins une demi-journée, pour visiter la médina de Fès.

10. Propos d'un guide touristique, syndicat d'initiative de Fès, 2004. 
mode de vie - la famille, le mariage, la cuisine et notamment les différences entre la cuisine turque, grecque et arabe, la situation économique de la population, le système social - et de la religion, en particulier l'Islam. D'autres questions portent sur la langue turque et certaines se réfèrent à l'histoire, à l'Empire ottoman, au sort du dernier sultan et surtout au harem. Parfois des questions abordent la situation politique du pays et les minorités kurdes et arméniennes.

Alors qu'à Fès les guides évoquent avec enthousiasme leurs échanges verbaux avec les touristes, les guides d'Istanbul, dans leur majorité, ont montré une attitude négative, voire méprisante, à l'égard des touristes et de leurs questions, que beaucoup qualifient de stupides. Si très certainement à Fès les relations entre les touristes et les guides ne sont pas exemptes de rapports de distinction, ceux-ci ne sont pas apparus de la sorte en entretien. A Istanbul, le rapport de distinction établi par le guide est clairement visible. A l'instar des observations d'Anne Doquet (2005) sur les représentations fines que les guides touristiques maliens associent aux touristes en fonction de leur nationalité, les guides d'Istanbul établissent une hiérarchie de valeurs en fonction de la nationalité des touristes. Il convient de préciser que les guides stambouliotes travaillent avec des groupes selon leurs compétences linguistiques: certains traitent uniquement avec des Japonais, d'autres avec des touristes anglophones, francophones ou encore des touristes russes. La hiérarchie ici établie est issue du croisement des entretiens avec les différents guides. Au bas de l'échelle se trouvent les Japonais «qui ne comprennent rien à rien et posent des questions absurdes sur la religion, le service militaire, la végétation ${ }^{11}$; certains manquent de culture générale selon les guides mais - au moins - ils n'ont pas de préjugés vis-à-vis des Turcs. S'y trouvent aussi les Américains qui posent des «questions absurdes ", portant sur le folklore et la vie quotidienne. Les touristes français et, par extension italiens et espagnols, sont considérés comme ayant des préjugés. Les Arabes, quant à eux, sont intéressés par les mosquées et font preuve d'intérêt pour la végétation. En haut de l'échelle, sont placés les Anglais, qui «posent des questions convenables, sur l'histoire ${ }^{12}$ et les Russes, qui "sont très cultivés, lisent beaucoup, connaissent l'histoire, en savent autant que le guide $»^{13}$.

11. Propos d'une guide travaillant avec des touristes japonais et exerçant depuis 1996, août 2005.

12. Propos d'un guide anglophone, août 2005.

13. Propos d'un guide russophone, août 2005. 
Les représentations des guides stambouliotes s'inscrivent dans une perspective plus générale de mépris dont sont communément affublés les touristes, «idiots du voyage » (Urbain 1991). Selon cette hiérarchie établie et face au manque de connaissances de certains touristes, on peut se demander si la distinction ne s'effectue pas en fonction du capital culturel, entre la connaissance des guides, qui ont une formation universitaire, et «l'inculture » - selon les guides - des touristes, alors que pour certains guides seuls les érudits sont jugés dignes d'intérêt ${ }^{14}$.

Cette distinction entre les questions absurdes et pertinentes des touristes révèle que toutes celles liées au folklore, aux coutumes populaires et à la ruralité sont jugées ineptes, alors que les questions portant sur l'architecture ou l'histoire semblent intéressantes. Est alors jugé recevable tout ce qui peut alimenter une culture urbaine et la grandeur de la Nation mais irrecevable ce qui rappelle la culture rurale et populaire. La distinction s'établit, à un autre niveau, entre l'Occident et l'Orient et les présupposés orientalistes. Enfin, l'ensemble des guides a dit refuser de répondre aux "questions désagréables ", qui concernent la politique et les minorités, peu compatibles avec l'image lissée et dépolitisée de la Turquie promue par l'économie touristique. Finalement, les questions problématiques sont celles qui renvoient à des projections identitaires équivoques, qui soulèvent le problème des minorités, associent la Turquie à un pays d'Orient ou évoquent les traces d'une culture anatolienne ou rurale à Istanbul.

\section{Jouer ou recadrer les stéréotypes}

Qu'en est-il alors du rôle de médiateur du guide ? Paradoxalement, on peut se demander comment les guides à Istanbul envisagent leur rôle de "passeurs » de connaissances alors qu'ils stigmatisent certains groupes de touristes et se montrent réticents vis-à-vis de la transmission de faits du quotidien, pourtant centraux dans l'expérience touristique.

14. Le niveau d'études des guides à Istanbul est sensiblement supérieur à celui des guides de Fès. Mais dans les deux cas ils sont diplômés de l'université, certains ont mené des cursus spécialisés en tourisme, ont passé le concours de guide à Fès et ont suivi la formation du ministère de la Culture et du Tourisme en Turquie s'ils ne sont pas passés par un institut spécialisé. Parmi les guides stambouliotes interrogés, figurent aussi un journaliste et un docteur en civilisation turque. Au demeurant, on ne peut occulter l'interaction de l'entretien, ayant spécifié aux guides que nous les interrogions dans le cadre d'une recherche doctorale. 
A Fès, on peut supposer que le dialogue impliquant le touriste et son guide affine la compréhension que le second a du premier. Selon la majorité des guides interrogés, les touristes, en venant à Fès, recherchent le dépaysement, quelque chose qui n'existe plus en Occident. Les touristes sont en quête de «la tradition ». A la suite des entretiens menés auprès des guides, il apparaît qu'ils se servent des clichés associés à la médina, réitèrent des discours orientalistes et jouent des traits saillants de l'identité locale promus dans la littérature touristique. Outre une rhétorique orientée sur le mystère de la ville et axée sur son aspect labyrinthique, se met en place une dialectique jouant sur les antinomies du type sombre/lumineux, ruelles étroites/ vastes demeures, transport à dos d'âne d'un téléviseur etc. Ils élaborent ainsi un discours spécifiquement destinés aux touristes, qui ne recoupe qu'en partie leurs représentations patrimoniales, plus nuancées ${ }^{15}$.

A Istanbul, plutôt que de s'inscrire dans la continuité des représentations des touristes sur la ville, les guides tentent de les infléchir. Par rapport au rôle qu'Erik Cohen a attribué aux guides professionnels (1985, 2001), la position des guides stambouliotes est relativement ambivalente. En effet, s'ils fournissent des informations précises sur les sites, ils ne paraissent pas les interpréter en fonction des attentes touristiques tandis que, dans un même temps, ils masquent certaines réalités urbaines, notamment au sujet de l'immigration. Ainsi, au risque d'aller à l'encontre de l'authenticité recherchée par les touristes, certains guides insistent sur les présupposés - sur la population turque, sur l'Orient et la modernité - qu'ils s'attachent à déconstruire et refusent d'engager des contacts plus que nécessaires. D'autres, au-delà de leur jugement de valeurs, s'attachent à réorienter les représentations des touristes tout en tentant de créer une atmosphère propre à l'enchantement. La difficulté est alors de briser les poncifs associés à la Turquie et réorienter l'image des touristes sur les Turcs, tout en réussissant à susciter l'enthousiasme et à maintenir un climat d'intimité. Lorsqu'il s'agit de créer un tableau jouant de l'ancien statut d'Istanbul, capitale de l'Empire ottoman, les guides alimentent, par leurs propos, l'imaginaire des touristes. En revanche, tout élément qui peut rappeler l'« anatolisation » d'Istanbul ou sa « ruralisation » est déconstruit. On valorise une culture urbaine. L'attitude des guides rejoint celles des "vieux stambouliotes »

15. Dans une précédente recherche, nous avons montré les écarts entre le discours patrimonial produit par le guide et ses propres représentations patrimoniales (Girard 2006). 
et des élites urbaines qui voient dans la prolifération de l'esthétique anatolienne un effet néfaste de l'exode rural (Fliche 2003).

A un autre niveau, les guides peuvent s'attacher à renverser certains préjugés relatifs à l'Islam ou à des modes de vie traditionnels et créer une proximité avec le touriste à partir d'éléments de la vie quotidienne « moderne » et compréhensible, offrant des similitudes avec les touristes européens. L'exemple de ce guide à propos des touristes français est éclairant :

Je sens bien que les gens ne connaissent pas grand-chose sur l'histoire. J'essaie pourtant de casser l'image du Turc. Car il s'agit «d'un Turc », pour les touristes ordinaires, l'image du Turc est bien définie, [...] le foulard. J'ai travaillé avec des Français du groupe Marmara et j'ai remarqué qu'il y a une grande réticence vis-à-vis des Turcs. Mes collègues me disaient que les Français insistaient sur le problème du foulard car il y a les médias et des débats en France. Je ne dis pas qu'ils ont des préjugés mais quand même. La question du foulard arrive au premier rang, la question de la montée de l'Islam en Turquie, la façon de percevoir la religion en Turquie sont les questions les plus posées [....]. Pour créer une atmosphère, cela dépend des cas. J'aime bien faire des comparaisons. Je compare les permis de conduire français et turc. Le permis de conduire turc a un style américain avec le groupe sanguin, le don d'organe. Pour une présentation, je prends l'exemple de la récupération des organes. On peut dire que le cœur est pris, que les reins ne servent à rien car je bois du raký... De temps en temps, je reçois des questions intéressantes comme «si la personne en question n'est pas morte, que faites-vous? », je réponds « on l'achève ». Des petites choses pour avoir des contacts plus humoristiques ${ }^{16}$.

Par ailleurs, si les guides proposent une autre version de "l'authenticité froide ", ils s'ingénient à répondre au désir d'« authenticité chaude » des touristes, plusieurs essayant de leur faire rencontrer la population. De plus, le dépaysement de la Turquie pour les touristes est à géométrie variable et, par conséquent, l'effort du guide pour susciter l'enchantement peut être plus ou moins marqué. Comme le note ce même guide,

ce que j'ai constaté, c'est qu'il y a des touristes qui sont plus impressionnés par les commentaires du guide, d'autres moins impressionnés. Pour les Japonais, la Turquie est peut-être plus exotique

16. Entretien avec un guide parlant français et japonais, journaliste, exerçant parallèlement la profession de guide depuis 1987, Chambre des guides touristiques d'Istanbul, août 2005. 
que pour les Français. Les Japonais sont très enthousiastes alors que pour les touristes européens, on est vraiment dans l'obligation de créer cette atmosphère enthousiasmante. On fait une gestuelle. Cela, c'est une autre chanson ${ }^{17}$.

Deux facettes du rôle du guide se dessinent donc dans l'expérience touristique : à Fès il conforte les touristes dans leur vision de l'Orient, tandis qu'à Istanbul il peut être tenté de l'infléchir. Pour ce faire, il prendra appui sur d'autres ressorts de la vie quotidienne pour susciter l'enthousiasme. Ces deux attitudes conduisent à formuler l'hypothèse qu'à Fès, le guide élabore un discours spécifiquement destiné à l'autre tandis qu'à Istanbul, il semble chercher à imposer ses propres représentations patrimoniales.

\section{Lartisanat, figure équivoque de la mise en scène de l'authenticité}

L'exemple de la mise en scène de l'artisanat illustre ces deux attitudes des guides dans la fabrication de l'authenticité : à Fès, l'artisan devient une figure phare de la médina vendue aux touristes; à Istanbul, la patrimonialisation et la mise en scène de l'artisanat sont l'objet de représentations équivoques.

La patrimonialisation et la mise en tourisme de l'artisanat se sont réalisées dans des temporalités et des contextes différents à Fès et Istanbul. A Fès, le statut patrimonial des artisans est depuis longtemps établi. La politique de revitalisation des arts indigènes menée par le Protectorat français a contribué à forger et surtout à médiatiser l'image de "Fès, capitale de l'artisanat »(Girard 2006). Aujourd'hui, l'artisanat apparaît comme une des figures phares de la médina, tant dans les discours exogènes, par exemple dans les guides touristiques français, que dans les images promotionnelles produites par les acteurs locaux.

A l'inverse, Istanbul se caractérise par la contemporanéité de la patrimonialisation et de la mise en scène de l'artisanat. Durant les années 1980, la demande touristique a nécessité de "se forger une image condensée de soi qui puisse être proposée aux visiteurs » (Sauner 2000 : 49). Dans ce contexte, les acteurs locaux s'emploient à façonner et à promouvoir des arts et artisanats traditionnels. Ces derniers renvoient aux artisanats manuels anatoliens tandis que les arts traditionnels deviennent un artefact patrimonial témoin du passé ottoman.

17. Id. 
A Fès, les lieux de l'artisanat deviennent des scènes de vie urbaine

Dans les espaces artisanaux, l'authenticité est ainsi mise en scène par le guide, dont le discours tend, comme le notait Erik Cohen (1985), à présenter de fausses informations comme étant vraies. Dans un même temps, il peut investir un rôle d'amuseur pour rendre la situation ludique. Les observations conduites place Seffarine et souk Nejjarine montrent que les guides touristiques encadrent des groupes allant de deux personnes à une quarantaine selon les cas. Leur passage ne dure que quelques minutes. Dans leurs discours, les guides insistent sur l'immuabilité des pratiques et l'ancestralité des savoir-faire. Par exemple, sur la place Seffarine qui regroupe des ateliers de chaudronniers et dont l'espace central est fréquemment utilisé pour le travail du cuivre et le stockage des marmites, les guides évoquent l'activité de la chaudronnerie et attirent l'attention sur le son des marteaux. Ils mentionnent aussi les « ragoûts », selon le terme qu'ils emploient, ce qui donne lieu à toutes sortes de digressions sur la cuisine. Le plus souvent, pour appuyer leur propos, ils touchent les objets, voire se saisissent d'un samovar, tout en pointant du doigt les artisans au travail. Les touristes, dont beaucoup rient lors de l'explication des guides, photographient les artisans tout en restant à quelques mètres de distance. Parfois aussi, ils touchent les produits. Les explications des guides sont donc stéréotypées. Elles ne tiennent compte ni des évolutions économiques ${ }^{18}-$ l'artisan étant cantonné dans un monde précapitaliste -, ni de l'évolution des produits fabriqués, forcément traditionnels, alors même que, par exemple, la plupart des menuisiers du souk Nejjarine confectionnent des sièges de mariée «modernes $»^{19}$. Cependant, comme l'a montré Nicolas Puig en se référant à l'analyse d'Yves Winkin, le guide doit initier l'euphorie, ce " moment privilégié de la découverte ", le « degré de réalisme de la description passant alors au second plan» (Puig 2000 : 74). En ce sens, on note une certaine convergence entre les guides et les acteurs publics autour de l'image à donner de la médina si l'on regarde les panneaux touristiques, installés dans le cadre du projet de sauvegarde de la

18. A partir des années 1970, un outillage mécanisé et des produits chimiques ont été introduits. La corporation a été en grande partie démantelée sous le Protectorat (Fejjal 1994). Pour une analyse des mutations sociales de la médina au XXe siècle, voir Berque (1972).

19. Ces sièges sont recouverts de plastique et agrémentés de fioritures en laiton, seule la structure est en bois. Leur esthétique comme leur forme se différencient des modèles traditionnels en bois. 
médina $^{20}$. Ces derniers mettent en scène l'histoire locale, à travers une réécriture axée sur le caractère immuable de la ville et sur la pérennité des pratiques artisanales. Par exemple, on peut lire dans le souk Nejjarine : «les artisans y produisent encore aujourd'hui du mobilier traditionnel en bois, témoin de la continuité des traditions ancestrales ».

L'artisanat mis en scène témoin de différentes interprétations de la culture à Istanbul

A Istanbul, la mise en scène de l'artisanat cristallise des approches plurielles de l'interprétation des désirs des touristes. Elle dévoile des rapports différenciés à la culture, entre la culture urbaine valorisée par les guides et les représentations de l'artisanat traditionnel portées par des acteurs publics ou encore mises en scène par les commerçants. Elle met ainsi au jour certaines ambivalences quant aux représentations de l'identité locale projetée et montre comment les guides peuvent être en porte-à-faux par rapport aux actions entreprises par d'autres acteurs locaux.

Lors des visites guidées, les espaces artisanaux occupent une place restreinte. Toutefois, certains guides amènent les touristes dans les centres artisanaux. Ces derniers, situés dans la péninsule historique, prennent place dans des medrese (écoles coraniques) datant de l'époque ottomane. Cette démarche s'inscrit dans un mouvement plus général de réinvestissement des bâtiments ottomans dans le contexte de la mise en tourisme d'Istanbul (Yenipehirlioðlu 1999). Dans les centres artisanaux, la mise en scène fonctionne sur le même mode. C'est l'esthétique ottomane qui est valorisée, bien qu'on puisse y trouver quelques éléments rappelant le décor anatolien. Ce sont essentiellement des arts traditionnels, tels que la calligraphie, la miniature, qui sont exposés. Tout est théâtralisé afin que le visiteur les identifie à l'époque ottomane. Lors de la visite, soit les guides fournissent des explications, soit ils laissent les artisans s'exprimer, une partie d'entre eux étant anglophones. Mais les guides peuvent aussi «simplement» amener les

20. Dans le cadre de la sauvegarde de la médina de Fès, six circuits touristiques, orientés selon des thématiques dont une sur l'artisanat, ont été créés. Le projet a été achevé en 2005. Celui-ci visait à décongestionner les circuits existants, à augmenter la fréquentation touristique et à favoriser les investissements dans la restauration des monuments sur les circuits aménagés. Sur les objectifs de ce projet, voir Hassouni et Serrhini (2004). 
touristes pour «boire un café, à cause de l'ambiance $»^{21}$. Dans ce cas, l'atmosphère doit suffire à provoquer l'enchantement sans que le guide ait à créer de discours. D'ailleurs, ici l'exposition des artisans n'est pas tant l'unique focale de la mise en scène qu'un élément d'un décor plus général devant susciter l'enchantement.

Or, les références à l'Anatolie, qui mettent notamment en scène la ruralité et qui se sont multipliées depuis les années 1990 ou encore les manifestations ponctuelles des artisanats manuels soutenues par les pouvoirs publics apparaissent en contradiction avec l'authenticité fabriquée par les guides. Témoin du refus des débordements sur l'espace public et des traces de l'Anatolie à Istanbul, les guides ont, par exemple, publié dans la revue de la Chambre des guides touristiques une lettre de protestation contre l'exposition des métiers manuels, organisée par le ministère de la Culture et du Tourisme, tenue en juin 2005 devant la basilique Sainte-Sophie. Ils ont ainsi argué que « ce festival n'avait pas bien assimilé la culture urbaine ", que l'endroit était mal choisi et que ce type de manifestation faisait perdre leur temps aux touristes ${ }^{22}$.

En outre, les guides peuvent être concurrencés par les commerçants, qui endossent eux aussi un rôle de médiateur culturel en mettant en scène la fabrication de tapis. A l'instar de ce qui existe depuis quelques années dans les restaurants avec les cuisinières anatoliennes (Sauner 1998), des tisseuses confectionnant des tapis sont installées en vitrine de magasins. La mise en scène est clairement signifiée comme une démonstration. La tisseuse est présentée comme une tisseuse anatolienne, c'est-à-dire vêtue d'un fichu, d'une jupe et de toutes sortes d'artifices rappelant le monde rural. Mais, la tisseuse tisse un tapis d'Hereke, c'est-à-dire un tapis ottoman d'inspiration impériale. Ainsi, les images produites s'appuient à la fois sur la ruralité et le faste ottoman. A l'intérieur du magasin, la situation est prise en main par le commerçant qui donne de nombreuses explications sur la fabrication du tapis, les conditions de vie des tisseuses anatoliennes, les types de tapis etc.

L'artisanat donne à voir deux visages du guide et de son rôle de médiateur culturel. A Fès, les guides, en tout cas ceux que nous avons

21. Propos d'un guide professionnel francophone, guide depuis 1975 , et travaillant avec des groupes sur des circuits et des voyages culturels à Istanbul, Izmir et en Cappadoce, août 2005.

22. Note interne affichée à la Chambre des guides d'Istanbul, datée du 26 juin 2005. 
observés, peuvent s'apparenter à la figure du guide professionnel (Cohen 1985) et apparaissent comme des inventeurs de la tradition. De façon significative, les guides sont souvent stigmatisés dans le discours des artisans : ils sont perçus entre autres comme des affabulateurs, construisant des discours fantaisistes. A Istanbul, les guides apparaissent comme des «équilibristes " (Chabloz et Raout 2009) : ils valorisent certains aspects de la culture et tentent d'en occulter d'autres, en particulier les signes de l'anatolisation, par ailleurs mis en scène par d'autres acteurs publics et privés. Les guides semblent alors être en porte-à-faux avec la quête d'authenticité des touristes.

\section{Authenticité des uns et des autres : retour sur les perceptions des touristes}

En retour, comment l'authenticité, « chaude » et «froide » (Selwyn 1996), fabriquée par les guides est-elle perçue par les touristes? En quoi l'imaginaire des touristes et les émotions ressenties lors de la visite de la ville, que l'on peut analyser à travers le concept «d'authenticité projective » (Saïdi 2010), convergent ou se distancient de l'imaginaire touristique véhiculé par les guides? Les sensibilités des touristes ne sont pas unes tandis que les représentations sont nuancées en fonction des motivations, du mode et de la durée de la visite et de l'origine des voyageurs.

L'authenticité, au regard des impressions des touristes, s'incarne dans les traces matérielles, les éléments immatériels qui participent des ambiances urbaines et dans la rencontre avec l'autre. L'émotion des touristes s'inscrit dans une relation à la temporalité, mais relativement différente à Fès et Istanbul puisque renvoyant à l'atemporalité dans le premier cas, à l'historicité dans le second. A Fès, plus que la profondeur historique de la médina prédomine l'idée d'une ville atemporelle, moyenâgeuse. Les expressions « Je n'ai pas seulement fait la visite de la médina, j'ai découvert une autre époque. C'est le Moyen Age », «Il y a des gens qui vivent comme au Moyen Age, comme cela était il y a 400 ou 500 ans ${ }^{23}$, "Elle est restée authentique, elle n'a pas bougée depuis 500 ans ${ }^{24}$, sont revenus dans l'ensemble des propos.

23. Propos de touristes français faisant un voyage organisé, hôtel Volubilis, mars 2004.

24. Propos de touristes français (un couple et le père du mari), hôtel Volubilis, mars 2004. 
«L'atemporalité » imaginée de la médina permet à la fois un déplacement dans le temps pour le touriste mais aussi dans l'espace alors que l'évocation du Moyen Age renvoie ici à l'image du Moyen Age européen. Les propos de ce touriste en sont une illustration : "C'est l'histoire européenne aussi. L'histoire de l'Europe, comment elle a été, le Moyen Age en Europe. C'est comme un retour aux sources ${ }^{25}$. L'unicité d'Istanbul provient quant à elle de sa grandeur historique, où des traces des empires byzantins et ottomans sont visibles, de sa place dans le monde musulman et de sa beauté. Elle est apparentée à la capitale ottomane. Istanbul impressionne par ses monuments, témoins de l'héritage ottoman et de l'identité islamique de la ville. Toutefois, l'imaginaire varie en fonction du lieu d'origine des touristes : les visiteurs venant d'Europe de l'Est ont plutôt tendance à rechercher les traces du syncrétisme religieux et de l'orthodoxie. Par ailleurs, la dimension paysagère ne s'inscrit pas uniquement dans un rapport à l'histoire. En effet, les touristes arabes et iraniens construisent, en partie, leur imaginaire autour des éléments naturels, notamment la mer.

Ces variations de l'imaginaire montrent en outre que l'émotion peut provenir du dépaysement mais aussi d'une histoire partagée dont le touriste recherche les traces. Le dépaysement s'incarne dans le contraste, notamment entre tradition et modernité. Par exemple, à Fès, la médina et la ville nouvelle sont opposées. A un autre niveau, la ville occidentale « aseptisée » est opposée à la médina, par déduction orientale, «vivante» et «authentique». A Istanbul, les évocations du contraste entre la tradition et la modernité, l'Orient et l'Occident, par ailleurs largement médiatisées par la littérature touristique, sont nombreuses chez les touristes interrogés sans pour autant que ces derniers ne s'éloignent du centre historique. Toutefois, selon les guides, la modernité de la ville, qui tient une place importante dans les impressions des touristes, peut susciter leur étonnement : «pour les Européens, c'est très différent de ce qu'ils veulent voir. Et pour les Orientaux, imprégnés de l'Empire ottoman, ils sont très étonnés de voir une ville occidentale ${ }^{26}$. Ces différentes appréhensions de l'Orient et de l'Occident témoignent du décalage entre ce que les touristes s'attendent à voir et ce qu'ils voient mais aussi décident de voir. Par ailleurs, les ambiances sont un signe du dépaysement. Tant à Fès qu'à Istanbul, les

25. Id.

26. Entretien avec un guide touristique francophone, août 2005. 
sons - celui du muezzin notamment -, et les odeurs - par exemple des épices - alimentent l'imaginaire des touristes, en leur fournissant des cadres de perception de la vie urbaine.

La « rencontre avec l'autre » tient une place centrale chez les touristes occidentaux. Les différentes perceptions montrent comment celle-ci renvoie à un dépaysement mais aussi à un effet de miroir sur le passé occidental. Elle peut aussi inscrire l'expérience dans un régime de partage d'une réalité contemporaine ou tout du moins perçu comme tel, un touriste interrogé à Fès soulignant que « la vieille ville n'est pas un musée. C'est des gens qui vivent, donc on vit avec eux ${ }^{27}$. Mais, à Istanbul, pour les touristes régionaux ou venant de pays du Sud, la rencontre avec l'autre peut renvoyer à une quotidienneté peu en phase avec l'expérience touristique.

A Fès, dans le regard des touristes, l'artisanat tient une place importante. L'ensemble des personnes interrogées a "vu » des artisans. Nous employons le verbe "voir» car, dans beaucoup de cas, la " rencontre », évoquée comme telle, se limite à un contact visuel. Toutefois, la visite des lieux de l'artisanat permet d'aborder les modes de vie et de créer une illusion de proximité avec un monde " inconnu » et «vrai ». En effet, selon certains, « cela permet de voir quelque chose qui n'existe plus en Occident ${ }^{28}$; « quelque chose qu'on ne voit plus en France et, quand on le voit, c'est uniquement touristique désormais. Tandis que là, on voit que les gens l'utilisent ${ }^{29}$.

Les touristes mettent en avant les savoir-faire, jugés de qualité, et les conditions de travail, jugées éprouvantes. De plus, en termes de représentations patrimoniales, tous les métiers sont englobés sous une même étiquette, que les techniques de fabrication aient été ou non modernisées.

A Istanbul, la rencontre avec l'autre se focalise sur la figure des danseuses, les vendeurs de kebabs, la population d'origine rurale, les vendeurs ambulants. Une touriste allemande note :

27. Propos d'un couple de touristes français, café Bâb Boujeloud, avril 2004.

28. Entretien avec un groupe de touristes allemands, restaurant Bâb Boujeloud, mai 2004.

29. Entretien avec une touriste française, aéroport de Fès-Saïss, mai 2004. 
C'est très agréable de s'asseoir là (place Sultanahmet). C'est très inspirant. [...] En Allemagne, c'est très différent : on ne peut pas voir des gens vendant de la nourriture à l'extérieur, ici on peut acheter ça [un épi de maïs]. C'est très agréable de sortir et de voir des gens qui vendent tout et...c'est très agréable de s'asseoir au milieu des gens ${ }^{30}$.

Par ailleurs, à Istanbul, les artisans ne sont pas évoqués spontanément, même si les lieux d'artisanat sont parfois visités. Des touristes interrogés ont dit être intéressés par les démonstrations dans les centres artisanaux. Comme l'évoque un guide interrogé, « les touristes sont très contents, c'est tout ce qu'ils cherchent, tout là-bas a un passé de 400 ans ${ }^{31}$. Ils mentionnent aussi la mise en scène des tisseuses anatoliennes. Les observations montrent d'ailleurs que les touristes peuvent être particulièrement friands de cette projection exotique. En outre, la réflexion d'une touriste, précisant «j'ai vu les métiers usuels mais pas les métiers typiques ${ }^{32}$, laisse supposer une hiérarchie entre les métiers qui auraient un intérêt patrimonial et touristique ou non. On est dans une projection quasi contraire à celle de Fès, où la «banalité du quotidien » était jugée « authentique » et par là même digne d'un intérêt touristique. En outre, les touristes bulgares et libyens ont fait part de leur désintérêt, chacun précisant venir de pays où l'artisanat est dynamique et les artisans nombreux ( «en Lybie nous avons la même chose ${ }^{33}$; « il y en a déjà beaucoup en Bulgarie ${ }^{34}$ ). Ici, ils ne sont alors plus confrontés à un quotidien exotique mais renvoyés à leur propre quotidien.

Les impressions des touristes ne sont pas uniques et selon l'endroit, la sensibilité esthétique varie. L'architecture, image forte d'Istanbul, n'est que secondairement mentionnée à Fès. De la même façon, la place de l'artisanat n'est pas identique: centrale à Fès, celle-ci apparaît ténue à Istanbul. Et l'imaginaire véhiculé et mis en scène par les guides ne paraît que relativement concordant avec les impressions des touristes et leur quête d'altérité. A Fès, les touristes évoquent souvent les images

30. Entretien avec une touriste allemande, voyageant seule et hébergée chez un ami, juillet 2005.

31. Entretien avec un guide touristique anglophone et hispanophone, août 2005.

32. Entretien avec une touriste italienne, voyageant en Turquie avec une amie, juillet 2005.

33. Entretien avec trois touristes libyens, août 2005.

34. Entretien avec six touristes bulgares, août 2005. 
stéréotypées associées à l'Orient. D'ailleurs, la visite orchestrée par les guides de la place Seffarine et du souk Nejjarine et plus généralement des lieux artisanaux suscite le plus souvent l'enchantement des touristes. La réussite semble provenir de l'effet de réel et de la mise en situation du guide. Toutefois, on peut se demander si les touristes sont dupes du discours des guides. En effet, l'image d'un artisanat immuable véhiculée par ces derniers semble relever d'une approche simplifiée, voire simplificatrice, de la sensibilité des touristes. A Istanbul, l'écart est plus patent, entre les convergences autour de l'émotion suscitée par la charge historique de la ville et sa monumentalité et les contrariétés alors que la population rurale, les petits métiers et l'esthétique anatolienne émeuvent nombre de touristes.

En conclusion, il convient de reposer la question du rôle mystificateur du guide et des émotions vécues par les touristes. A la première question, l'analyse comparée des cas de Fès et d'Istanbul appelle une réponse nuancée. A Fès, l'authenticité fabriquée par le guide s'appuie sur les stéréotypes associés à la médina et plus généralement à l'Orient. Ils sont en ce sens des passeurs d'une tradition qu'ils contribuent à inventer et des acteurs centraux du processus d'orientalisation de la médina. Si à Fès le dialogue entre le touriste et son guide contribue à rendre le discours de ce dernier plus performatif, à Istanbul il semble plutôt témoigner d'un rapport de distinction. Celui-ci s'exprime dans la différenciation établie entre les questions des touristes, jugées re- ou irrecevables, pertinentes ou ineptes, et dans la typification des touristes qui en résulte. Au regard de la figure du « guide professionnel » (Cohen 1985, 2001), les guides stambouliotes ne jouent que partiellement le jeu. En effet, ils contrecarrent la quête d'authenticité des touristes ou cherchent à faire évoluer leurs perceptions quand celles-ci reposent sur des représentations identitaires équivoques renvoyant à une image caricaturale de l'Orient, à « l'anatolisation » d'Istanbul, à la question des minorités en Turquie. Ainsi, les guides semblent tentés d'imposer leurs propres représentations et, finalement, de ramener la scène en coulisse. Et, si les guides proposent d'autres versions de l'authenticité, elles ne prennent en compte et ne reflètent qu'en partie les réalités contemporaines. Est alors souligné ce qui renvoie à la modernité et à l'occidentalité de la ville et de la société turque mais occulté ce qui ne correspond pas à la culture urbaine valorisée.

L'attitude contrastée des guides à Fès et Istanbul témoigne d'un rapport différencié à la culture - de distanciation dans le premier cas et 
de crispation identitaire dans un contexte de réappropriation du passé ottoman et de fortes mutations urbaines dans le second - que traduit la mise en scène de l'artisanat. Ainsi, à Fès, la fabrication de l'authenticité s'appuie sur des images décontextualisées de l'artisanat, renvoyant à la tradition et à l'ancestralité, par ailleurs relayées par les pouvoirs publics. A Istanbul, en opérant une distinction entre les arts traditionnels, évocations de l'Empire ottoman et d'un passé fastueux, et l'artisanat manuel anatolien, jugé incompatible avec l'identité de la ville, les guides apparaissent en porte-à-faux avec d'autres représentations de la culture proposées par les acteurs locaux de la mise en patrimoine et en tourisme, ainsi qu'avec une partie des touristes.

Ainsi, à Istanbul mais aussi dans une certaine mesure à Fès, l'imaginaire touristique véhiculé par le guide ne converge qu'en partie avec l'expérience vécue des touristes, fondée sur des émotions multiples. L'émotion résulte, à l'instar de ce qu'a montré Habib Saïdi, de "l'éloignement dans l'espace et le temps [qui] est l'un des facteurs déterminants de l'accomplissement de soi lors d'une expérience touristique »(Saïdi 2010 : 471). Elle résulte aussi, selon l'origine des touristes, de la recherche d'une histoire partagée. Enfin, la place de la rencontre avec l'autre dans l'expérience touristique, dont l'artisanat est un témoin fort à Fès, secondaire à Istanbul, est à géométrie variable : centrale pour les touristes occidentaux, elle semble renvoyer pour les touristes régionaux et des pays du Sud à une quotidienneté peu propice à l'émotion.

L'attitude contrastée des guides à Fès et Istanbul tout comme les multiples impressions des touristes invitent, dans une perspective future, à observer d'autres types de guides, comme les faux guides, afin d'éclairer le caractère pluriel de l'authenticité fabriquée. Elle engage aussi à se centrer sur les touristes du Sud pour proposer une autre lecture des ambivalences identitaires liées au tourisme. Ce regard décentré apporterait un autre éclairage sur la question de l'imaginaire touristique et de l'authenticité. 


\section{Références}

Adner, Marie et Evelyne Bastin. 2000. Artisanat et tourisme dans la région de Fès-Boulemane. I.U.P Aménagement et Développement Territorial, Institut de Géographie Alpine de Grenoble.

Bartu, Ayfer. 2001. "Rethinking Heritage Politics in a Global Context : a View from Istanbul ». In Nezr Al Sayyad (dir.). Hybrid Urbanism : On the Identity Discourse and the Built Environment: 131-155. Londres : Praeger.

Berque, Jacques. 1972. "Fès ou le destin d'une médina ». Cahiers internationaux de sociologie, LII : 5-32.

Brown, David. 1999. "Des faux authentiques. Tourisme versus pèlerinage ». Terrain, 33: 41-56 (Mis en ligne le 26 juillet 2005. URL : http://terrain.revues.org/document2713.html. Consulté le 16 avril 2006).

Chabloz, Nadège et Julien Raout. 2009. «Corps et âmes. Conversions touristiques à l'africanité ». Cahiers d'Etudes africaines, XLIX (193194) : 7-26.

Cohen, Erik. 2001. Thaï Tourism. Hill Tribes, Islands and Open-ended Prostitution. Bangkok: White Lotus Press.

—. 1988. "Authenticity and Commoditization in Tourism ». Annals of Tourism Research, $15: 371-386$.

— 1985. «The Tourist Guide : The Origin, Structure and Dynamics of a Role ». Annals of Tourism Research, $12: 5-29$.

Cravatte, Céline. 2009. «L'anthropologie du tourisme et l'authenticité. Catégorie analytique ou catégorie indigène ?». Cahiers d'Etudes africaines, XLIX (193-194) : 603-619.

Doquet, Anne, 2009. " "Guides, guidons et guitares". Authenticité et guides touristiques au Mali ». Cahiers d'Études africaines, XLIX (193194) : 73-94.

—. 2005. "Tous les Toubabs ne se ressemblent pas. Les particularités nationales des étrangers vues par des guides touristiques maliens ». In GEMDEV et Université du Mali (dir.), Mali-France : Regards sur une histoire partagée : 243-258. Paris : Khartala ; Bamako: Donniya.

Doquet, Anne et Sara Le Menestrel. 2006. "Introduction ». Autrepart, $40: 3-13$.

Eldem, Edhem. 2010. Un Orient de consommation. Istanbul : Musée de la Banque ottomane.

Fejjal, Ali. 1994. Fès : héritages et dynamiques urbaines actuelles. Thèse d'Etat en géographie, Université François Rabelais de Tours. 
Fliche, Benoît. 2003. Les vacances de Kayalar. Histoire migratoire, usages et représentations d'un village anatolien en milieux urbains (Turquie, France). Thèse de doctorat, Université d'Aix-en-Provence.

Girard, Muriel. 2010. Recompositions du monde artisanal et mutations urbaines au regard des mises en patrimoine et en tourisme au Maghreb et au Moyen-Orient (Fès, Istanbul, Alep). Thèse de doctorat, Université François Rabelais.

- - 2006a. "Invention de la tradition et authenticité sous le Protectorat au Maroc : l'action du Service des Arts indigènes et de son directeur Prosper Ricard». Socio-anthropologie, 19 : 31-46.

- 2006b. "Imaginaire touristique et émotion patrimoniale dans la médina de Fès (Maroc) ». Culture et Musées, 8: 61-90.

Hassouni, Omar et Fouard Serrhini. 2004. «Fès ». In Patrimoine et développement durable dans les villes historiques du Maghreb. Enjeux, diagnostics et recommandations: 137-142. Rabat: Bureau de l'UNESCO.

Lahbil-Tagemouati, Naïma. 2001. Dialogue en médina. Casablanca: Editions du Fennec.

MacCannell, Dean. 1976. The Tourist. A New Theory of the Leisure Class. Londres : Basingstoke.

—. 1973. "Staged Authenticity: Arrangement of Social Space in Tourist Settings ». American Journal of Sociology, 79 (3) : 589-603.

Michaud, Jean. 2001. "Anthropologie, tourisme et sociétés locales au fil des textes ». Anthropologie et sociétés, 25 (2) : 15-33.

Nash, Dennison. 1981. «Tourism as an Anthropological Subject». Current Anthropology, 22: 461-481.

Otmani, Rachid. 1985. Les impacts économiques et sociaux du tourisme international sur l'artisanat de la médina de Fès. Thèse de doctorat en sociologie, Université Paris VIII, 2 vol.

Pérouse, Jean-François. 2003. «La question du patrimoine à Istanbul : le cas de la muraille terrestre ». Rives nord-méditerranéennes, 16 : $27-44$.

- 2004. La Turquie en marche: les grandes mutations depuis 1980. Paris : Editions de la Martinière.

Poulot, Dominique. 2006. «Introduction. Défendre le patrimoine, cultiver l'émotion ». Culture et Musées, 8: 13-25.

Puig, Nicolas. 2000. «Entre souqs et musées ». Espaces et sociétés, 100 : 57-80.

Reisinger, Yvette et Carol Steiner. 2006. «Reconceptualizing Object Authenticity ». Annals of Tourism Research, 331 (1) : 55-86. 
Saïdi, Habib. 2010. «Touristes québécois en Tunisie et patrimoine du "soi authentique". Ethnologie française, XL 3: 469-476.

Sauner, Marie-Hélène. 1998. «Raviolis à la Topkapý ». Mediterraneans, $10: 444-447$.

- 2000. «La cuisine ottomane ou la transmission d'un art de vivre ». La pensée de midi, 3 : 45-51.

Selwyn, Tom (dir.). 1996. The Tourist Image : Myths and Myth Making in Tourism. Chichester: Wiley.

Urbain, Jean-Didier. 1991. L'idiot du voyage. Histoires de touristes. Paris : Plon.

Urry, John. 1990. The Tourist Gaze. Leisure and Travel in Contemporary Societies. Londres : Newbury Park, Sage.

Wang, Ning. 1999. "Rethinking Authenticity in Tourism Experience ». Annals of Tourism Research, 26 (2) : 349-370.

Winkin, Yves. 1998. «Le touriste et son double. Eléments pour une anthropologie de l'enchantement». In Susan Ossman (dir.). Itinéraires de soi et paysage de la rencontre: 133-143. Paris : Editions du CNRS.

Yenipehirlioðlu, Feliz Çalýplar. 1999. «Decontextualisation and Recontextualisation of Ottoman Cultural Heritage in Post-Ottoman Nation-States ». In Meral Korzay, Nedret Kuran Burçoðlu et al. (dir.). Heritage / Multicultural Attractions and Tourism : 119-142. Istanbul : Boðaziçi University. 